\section{Scientific rationale for extrapolation of biosimilar data across cancer indications: case study of CT-P10}

\author{
Michinori Ogura ${ }^{*, 1}$, Bertrand Coiffier ${ }^{2}$, Hyuk-Chan Kwon ${ }^{3}$ \& Sang Wook Yoon ${ }^{3}$
}

For a biosimilar to gain regulatory approval, a comprehensive comparability exercise must demonstrate that it is highly similar to its originator biologic, or reference product. Once biosimilarity has been shown, it is possible to approve the biosimilar for additional indications held by the reference product, without clinical trials in these indications. Extrapolation of clinical data is permitted by regulatory agencies as long as it is scientifically justified. CT-P10, a biosimilar of rituximab, was recently approved in Europe for all indications held by its reference product, incorporating both autoimmune diseases and hematological cancers. Here, we review the scientific rationale for extrapolation in biosimilar development using the example of CT-P10 as a case study.

First draft submitted: 30 March 2017; Accepted for publication: 20 April 2017; Published online: 9 May 2017

Biosimilars are highly similar versions of already licensed biologic products and represent a unique class of drugs requiring specific considerations for regulatory approval. Indeed, biosimilars undergo an expedited development and approval process that differs from that for novel or 'originator' biologics but that, nonetheless, are highly rigorous [1-6]. This process includes head-to-head comparisons of the biosimilar versus its reference product in all the stages of nonclinical and clinical evaluation that originator biologics pass through during their development, plus a comparative quality assessment. With respect to clinical trials, the nature and extent of the data required to approve an individual biosimilar depend on several factors, including: the level of evidence supporting biosimilarity in analytical, in vitro and, if performed, nonclinical in vivo studies; the occurrence of particular safety issues associated with the originator drug (or 'reference product') and the potential for extrapolation across indications. In this context, the term 'extrapolation' refers to the approval of a biosimilar for one or more additional indications held by the reference product, without the need for clinical trials of the biosimilar in those indications (Figure 1).

The general concept of extrapolation, for example from adult to pediatric patient populations or between drugs within the same class, is accepted as scientifically logical by key regulatory bodies, including the EMA and the FDA. In its concept paper on the extrapolation of efficacy and safety in medicine development, the EMA defines extrapolation as follows: "Extending information and conclusions available from studies in one or more subgroups of the patient population (source population), or in related conditions or with related medicinal products, to make inferences for another subgroup of the population (target population), or condition or product, thus reducing the need to generate additional

'Department of Hematology, Tokai Central Hospital, Gifu, Japan

${ }^{2}$ Hospices Civils de Lyon, Pierre-Benite, France

${ }^{3}$ CELLTRION Healthcare Co. Ltd, Incheon, Republic of Korea

*Author for correspondence: Tel.: +81 58382 3101; Fax: +81 58382 1762; mi-ogura@naa.att.ne.jp

\section{KEYWORDS}

- biosimilars • chronic lymphocytic leukemia - CT-P10 • diffuse large B cell lymphoma • extrapolation - follicular lymphoma - non-Hodgkin's lymphoma - rituximab - totality of evidence 


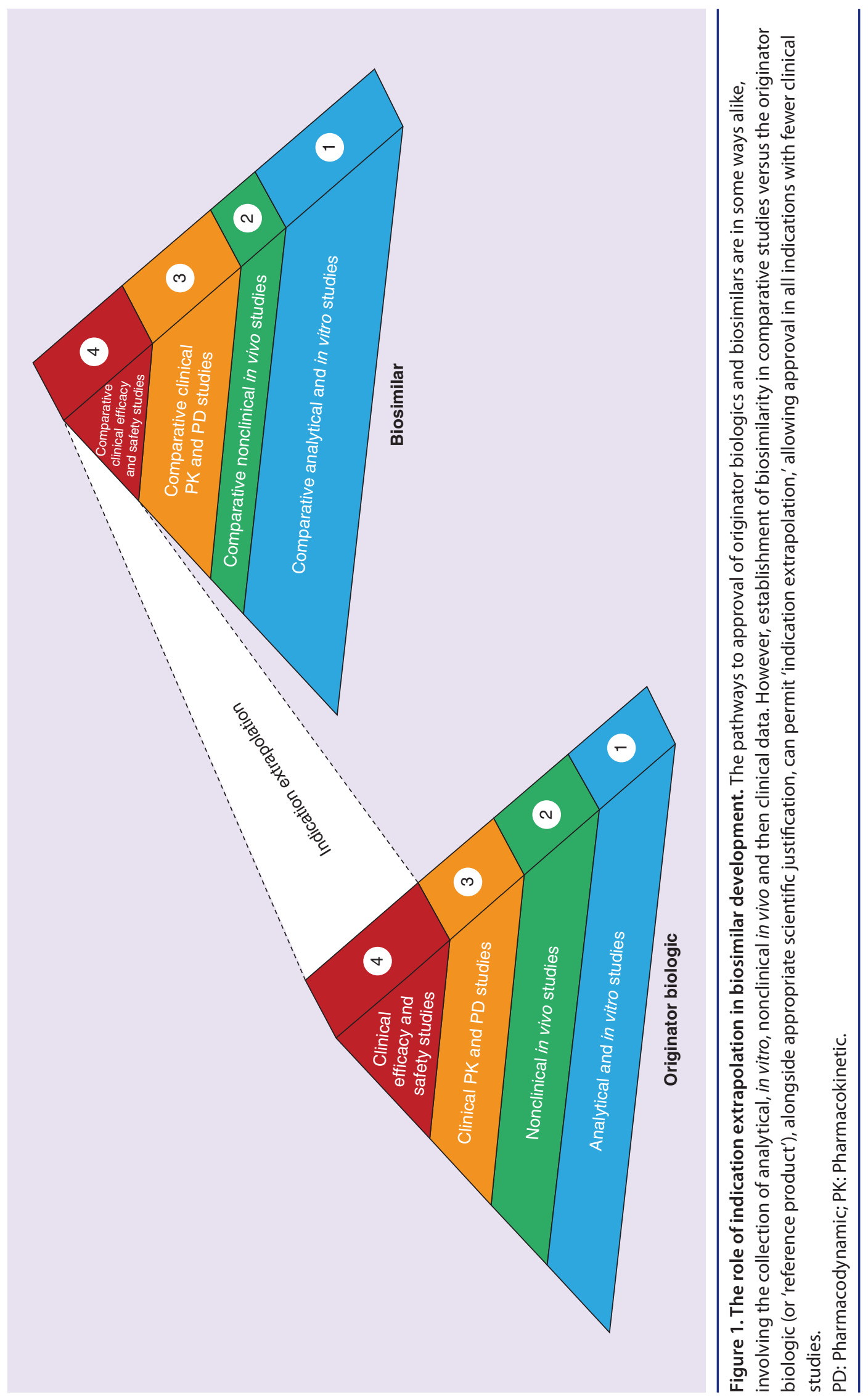


information (types of studies, design modifications, number of patients required) to reach conclusions for the target population, or condition or medicinal product" [7]. The EMA (and others) recognize that this process avoids unnecessary, and therefore unethical, clinical trials and encourages allocation of resources in areas where studies are most needed. For biosimilars, extrapolation reduces the burden of the development process, thereby potentially shortening the time to market and resulting in lower development costs that can be passed on in terms of reduced pricing. In this article, we discuss the scientific rationale for extrapolation in biosimilar development using the example of CT-P10 as a case study. For background, we begin by briefly discussing the general requirements of regulatory authorities with respect to extrapolation.

\section{When is extrapolation permitted?}

For approval of a biosimilar to be extrapolated across indications, regulators require: sufficient nonclinical and clinical data to prove biosimilarity to the reference biologic (often referred to as the 'totality of evidence'); and scientific justification for extrapolation from source-to-target populations [4-6]. In practice, this requires collection of extensive nonclinical data comparing the structure and function of the biosimilar and reference product, as well as the performance of randomized controlled trials in an indication (or indications) considered sufficiently sensitive to detect clinically meaningful differences in efficacy or safety between the biosimilar and its reference product (if such differences were to exist). Scientific justification for extrapolation is often multifaceted and nuanced but usually centers on demonstrating either that the mechanisms of action and clinical profile of the reference product are the same in the source and target populations, or why any such differences between source and target populations would not be expected to result in clinically meaningful differences between biosimilar and reference product in the target population(s) [4]. If the molecular mechanism of action of the reference product is different across indications, or the contribution of different mechanisms is unknown, additional pharmacological data will be required to justify extrapolation and provide assurance that the two products will behave in a similar manner in the extrapolated indications [1]. Furthermore, special consideration may be required to justify extrapolation of immunogenicity data as there are numerous influencing factors that can differ among indications, such as route of administration and concomitant medications [1].

\section{Approval of CT-P10 in Europe}

CT-P10 (Truxima ${ }^{\circledR}$; CELLTRION, Incheon, Republic of Korea), a biosimilar of the antiCD20 monoclonal antibody rituximab, was recently approved in Europe for all indications held by reference rituximab (MabThera ${ }^{\circledR}$; Roche, Basel, Switzerland), including non-Hodgkin's lymphoma (NHL) and chronic lymphocytic leukemia (CLL) [8]. Thus, CT-P10 became the first monoclonal antibody biosimilar therapy approved for use in hematological cancers (and in oncology in general). In NHL, CT-P10 is approved for use in combination with chemotherapy in previously untreated patients with stage III-IV follicular lymphoma (FL), as maintenance therapy in patients with FL responding to induction therapy, and in combination with CHOP chemotherapy (cyclophosphamide, doxorubicin, vincristine and prednisolone) in patients with CD20-positive diffuse large B cell lymphoma (DLBCL). It can also be used in combination with chemotherapy in treatment-naive and relapsed/refractory CLL. Non-oncological indications are rheumatoid arthritis (RA) and granulomatosis with polyangiitis (GPA) and microscopic polyangiitis (MPA) [8].

As we briefly discuss in the next section, approval of CT-P10 was based on the totality of evidence collected in analytical, in vitro and nonclinical in vivo experiments plus clinical data from randomized controlled trials in patients with RA and advanced FL. These trials demonstrated that there were no clinically meaningful differences between CT-P10 and reference rituximab (abbreviated hereafter as 'RTX') in these patient populations. Approval of CT-P10 in other indications held by RTX, including DLBCL and CLL, was based on extrapolation of data from these trials.

\section{Establishing the biosimilarity of CT-P10 \&} reference rituximab

A comprehensive three-way comparability exercise was performed to fully characterize the structural and physicochemical properties of CT-P10 relative to both US-sourced and EU-sourced RTX (Rituxan ${ }^{\circledR}$ [Genentech, CA, USA] and MabThera, respectively) [8,9]. These analytical studies demonstrated a high degree of similarity between CT-P10 and both forms 
of RTX [8]. In vitro biological and functional analyses also demonstrated comparable CD20binding affinities and complement-dependent cytotoxicity (CDC), antibody-dependent cellmediated cytotoxicity (ADCC) and apoptosisinducing activities $[8,10]$.

Randomized controlled studies compared the pharmacokinetics (PK), efficacy, safety and immunogenicity of CT-P10 and RTX in patients with RA and advanced FL [10-16]. Briefly, PK equivalence of CT-P10 and RTX was demonstrated in Phase I and III trials in patients with RA [10,12]. Efficacy profiles were also highly similar in this population, with the Phase III trial demonstrating therapeutic equivalence in terms of reductions in disease activity scores between baseline and week $24[10,13]$. The safety and immunogenicity profiles of RA patients treated with CT-P10 or RTX were also comparable $[10,12-13]$. B cell kinetics in patients with RA treated with CT-P10 or RTX were also shown to be similar: there was a reduction in median B cell count to below the lower limit of quantification at $4 \mathrm{~h}$ post-treatment, which remained low until the last measured end point at 24 weeks [10]. In patients with untreated advanced FL, the PK of CT-P10 and RTX was determined to be equivalent when each was given in combination with cyclophosphamide, vincristine and prednisone (CVP) chemotherapy [11]. Pharmacodynamic analyses up to treatment cycle 4 in this patient population showed that $\mathrm{B}$ cell levels decreased below the lower limit of quantification shortly after cycle 1 and subsequently remained low in both treatment groups. Safety and immunogenicity profiles up to cycle 4 were also comparable between groups [11].

Scientific rationale for extrapolation of CTP10 clinical data across indications

As we have described, extrapolation from one indication of a reference product to another in which the biosimilar has not been clinically tested is permitted if the totality of evidence supports biosimilarity and it is scientifically justified. In the case of CT-P10, the decision of the EMA to approve it for all indications held by its reference product was based on the totality of evidence showing biosimilarity between CT-P10 and RTX in nonclinical evaluations and in clinical trials in RA and advanced FL, as well as on the further scientific justifications described below [8].

\section{- Selection of source populations}

Selection of the patient populations recruited to CT-P10 clinical trials involved careful consideration. According to regulatory guidelines, biosimilars should be evaluated in indications that are sufficiently sensitive to detect clinically meaningful differences in efficacy or safety between biosimilar and reference product. As there is a long history of RTX use in RA [17], there were extensive clinical data with which to define and validate efficacy equivalence margins for the statistical comparison of CT-P10 with RTX; similarly, the history of use of RTX in FL provided sufficient data on which to predict efficacy in that population. Ultimately, RA and FL populations were considered adequately sensitive to detect meaningful differences between CT-P10 and RTX. The EMA also recommends that, for clinical studies assessing biosimilarity, the source population should be homogenous [5]. Therefore, the selection of FL as a source population for trials comparing CT-P10 and RTX in patients with cancer was considered particularly appropriate as, compared with the biologically and clinically diverse DLBCL for example, FL is a relatively homogenous disease.

\section{- Similarity in pathology between RA \& $B$ cell-related malignancies}

A significant factor that permitted cross-indication extrapolation for CT-P10 is the primary role of B cells in the pathogenesis of RA and certain hematological malignancies $[18,19]$. In RA, B cell checkpoints that monitor self-tolerance are defective, leading to the accumulation of autoreactive B cells that promote leukocyte infiltration into joints, causing inflammation of the synovial membrane and bone destruction $[18,20]$. In B cell-related hematological malignancies, defective apoptosis and altered survival signaling of B cells leads to their accumulation in the blood or lymph nodes [19].

\section{- Rituximab mechanisms of action}

RTX is a monoclonal antibody that binds with high specificity to CD20. This cell surface antigen is present on normal and malignant $\mathrm{B}$ cells during cell maturation and antigen-stimulated activation but absent on lymphoid stem cells and plasma cells [21,22]. The benefit of targeting CD20 in B cell malignancies was first realized almost three decades ago [23], and led to RTX becoming the first monoclonal antibody 


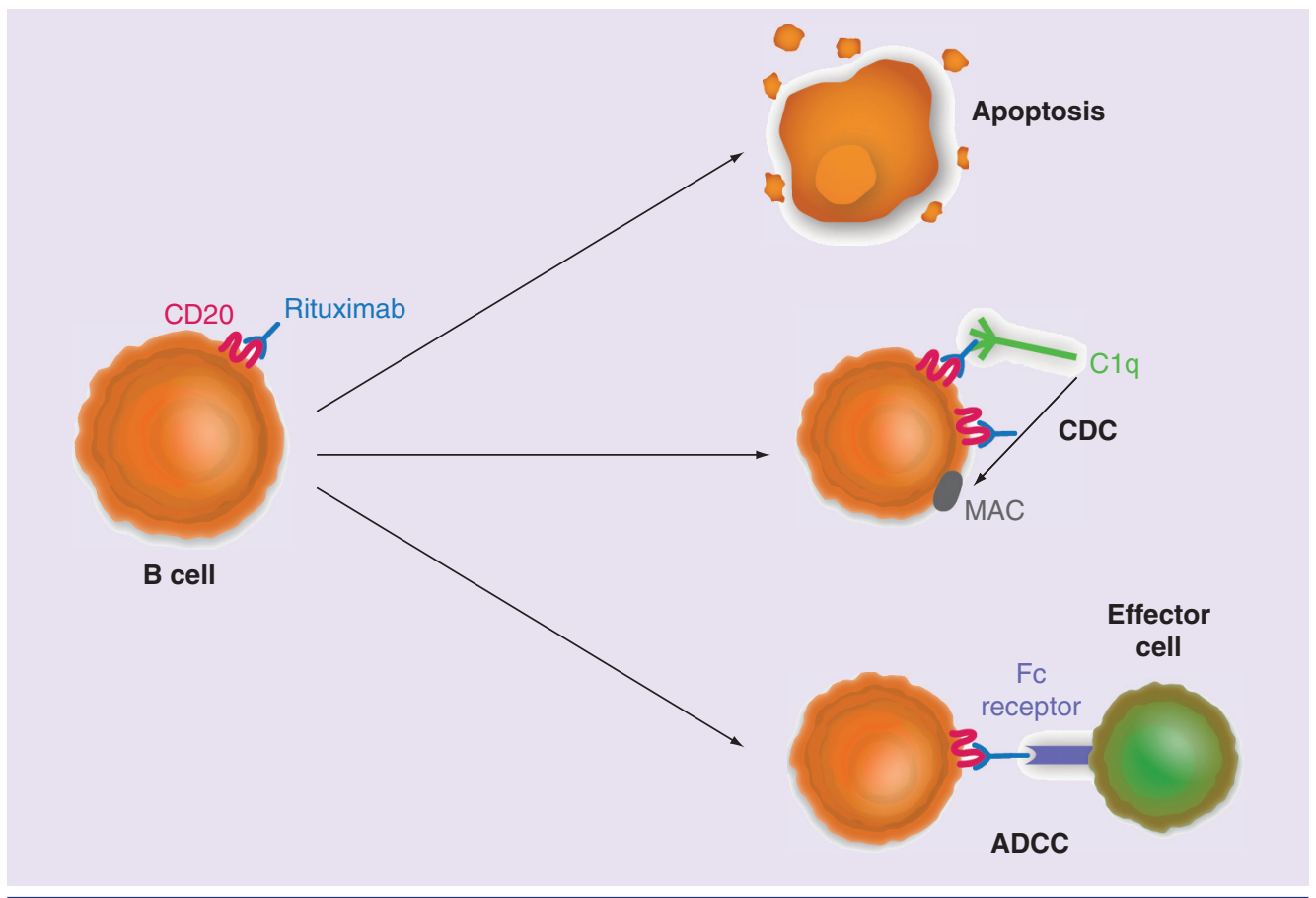

Figure 2. Main mechanisms of action of rituximab. Rituximab can deplete $B$ cells through direct induction of apoptosis and activation of the immune effector mechanisms CDC and ADCC. ADCC: Antibody-dependent cell-mediated cytotoxicity; CDC: Complement-dependent cytotoxicity; MAC: Membrane attack complex.

approved for the treatment of cancer in the USA in 1997 and Europe in 1998. Depletion of B cells by CD20-bound rituximab has primarily been attributed to CDC, ADCC and apoptosis (Figure 2) [22,24-25]. Comprehensive literature searches have not identified evidence suggesting a difference in the CD20-mediated effects of RTX between autoimmune and oncology indications. However, the clinical significance of each mechanism within each RTX indication remains to be determined. Importantly, in vitro functional analyses of CT-P10 have demonstrated that it has highly similar CDC, ADCC and apoptotic activity compared with RTX $[8,10]$. Analyses of functional end points, potency and binding affinity relating to apoptosis, CDC and ADCC conducted using samples from patients with NHL and CL also supported CT-P10 indication extrapolation to oncology indications from data obtained in RA [8].

\section{Complement-dependent cytotoxicity}

Rituximab is a type I CD20 monoclonal antibody, with in vitro analyses showing it can bind to the complement component C1q and trigger the classical complement pathway $[26,27]$. This capability is dependent on the redistribution of CD20 into lipid rafts within the target cell membrane, which is thought to make the cell more vulnerable to complement attack [26]. However, although some in vivo studies have indicated that this mechanism is important for the therapeutic effects of rituximab [28,29], others have found no evidence that complement is necessary for the drug's ability to deplete B cells [30]. Furthermore, the expression of complement inhibitors (CD46, CD55 and CD59) on tumor cells did not correlate with clinical outcome following rituximab treatment in patients with FL or CLL $[31,32]$. Therefore, although it is clear that rituximab can induce CDC, the importance of this mechanism in the therapeutic effects of the drug is not certain $[24,25]$. Furthermore, some evidence suggests that the CDC activity of rituximab is related to CD20 expression levels [33]. CLL cells are known to express lower levels of CD20 compared with FL, and it has been suggested that this may explain the poorer responses of CLL to rituximab [34]. This may not invalidate extrapolation between indications, however, as it is likely that other mechanisms (e.g., ADCC) may act in a complementary fashion [33]. 
Antibody-dependent cell-mediated cytotoxicity

ADCC depends upon recognition of the bound anti-CD20 antibody on the B cell surface by fragment crystallizable $\gamma$ receptors (Fc $\gamma \mathrm{Rs}$ ) present on natural killer cells [35]. These cells become activated through their Fc $\gamma \mathrm{R}$ and release cytotoxic granules to kill the antibodycoated CD20-positive cell. Evidence for the role of ADCC in the therapeutic activity of rituximab comes from correlations of receptor polymorphisms and drug responses. Genomic polymorphisms affect the affinity of Fc $\gamma \mathrm{R}$ for the Fc portion of the anti-CD20 antibody, and thus affect the killing activity of effector cells [36]. For example, a single nucleotide polymorphism in one form of the Fc $\gamma$ R (Fc $\gamma$ RIII) has been correlated with rituximab efficacy in FL [37] and RA [38]. This suggests the importance of Fc-mediated ADCC in effective therapy. However, the clinical relevance of such polymorphisms for rituximab immunotherapy on longer-term survival across B cell malignancies is still unclear, particularly when administered in combination with chemotherapy [39-41]. In particular, high-affinity variants were not associated with better responses in patients with CLL or DLBCL, suggesting other modes of action of rituximab such as CDC and apoptosis are more important in these cancers [39,41]. In vitro functional analysis has demonstrated that the FcRn-binding affinities of CT-P10 and RTX show almost identical equilibrium dissociation constants $[8,10]$.

\section{Cell signaling \& apoptosis}

Evidence suggests that direct ligation of CD20 by rituximab can impact intracellular signaling, leading to inhibition of antiapoptotic and survival signaling pathways [42]. This has been shown to induce apoptosis in CLL cells [43]. Inhibition of pathways, including the NF- $\kappa \mathrm{B}$, by rituximab has also been shown to sensitize lymphoma cells to cytotoxic drugs [44]. Further in vitro analysis that used a modified rituximab that lacked the $\mathrm{Fc}_{c}$ fragment determined that these rituximab-mediated effects were Fc-independent, without the need for FcR cross-linking, and occurred as a direct result of rituximab binding to CD20 [45]. Although these studies suggest a role for rituximab in promoting cell death, the contribution of this mechanism to the clinical therapeutic effects of rituximab across indications is still unclear.

\section{Conclusion}

Extrapolation is fundamental to the biosimilar concept. It efficiently reduces the number of clinical trials that are needed, which allows the regulatory process to be expedited. This can lead to price reductions compared with innovator biologics and potential savings for healthcare systems. The EMA-approved CT-P10 for all indications held by RTX based on the evidence of biosimilarity demonstrated in the comprehensive comparability assessment, which included clinical trials in patients with RA and FL. Extrapolation to CLL, GPA, MPA and DLBCL was scientifically justified based on the strength of clinical data in the source populations, the similarity of disease pathology across indications and the known mechanisms of action of rituximab in $B$ cell-related cancers and autoimmune diseases.

\section{Future perspective}

It is expected that postauthorization surveillance of CT-P10 will further support the EMA's decision to approve the biosimilar in all indications held by RTX. Such evidence will also provide confidence for clinicians, patients and healthcare providers in CT-P10 and biosimilars in general. As patent expiry of other biological therapies is reached, it is inevitable that these too will be followed by the approval and introduction of biosimilar versions into clinical practice. Strict adherence to published guidelines that recognize the importance of scientific justification will ease the potential safety concerns in cross-indication extrapolation, and will aid in the regulatory approval and market uptake of biosimilars.

\section{Financial \& competing interests disclosure}

$M$ Ogura has received research funding from Symbio, and personal fees from CELLTRION, Celgene, AstraZeneca, Takeda, Mundipharma, Janssen and Meiji Seika Pharma outside the submitted work. B Coiffier has received personal fees from Gilead, Celgene, CELLTRION, Roche, MorphoSys, MedImmune, Mundipharma and Pfizer outside the submitted work. H-C Kwon and SW Yoon are employees of CELLTRION Healthcare Co., Ltd. The authors have no other relevant affiliations or financial involvement with any organization or entity with a financial interest in or financial conflict with the subject matter or materials discussed in the manuscript apart from those disclosed.

Writing assistance was utilized in the production of this manuscript. Medical writing support during the preparation of this article was provided by Alice Wareham PhD (Aspire Scientific, Bollington, UK) and was funded by CELLTRION Healthcare Co., Ltd (Incheon, Republic of Korea). 


\section{EXECUTIVE SUMMARY}

\section{Background}

- Extrapolation refers to the approval of a biosimilar for one or more additional indications held by the reference product, without the need for clinical data in those indications.

\section{When is extrapolation permitted?}

- Extrapolation is permitted by regulatory agencies as long as it is scientifically justified.

\section{Approval of CT-P10 in Europe}

- CT-P10 is a biosimilar of rituximab, an anti-CD20 monoclonal antibody approved for use in autoimmune and cancer indications.

- CT-P10 was approved for all indications held by reference rituximab based on the totality of evidence collected in the comprehensive comparability exercise.

\section{Establishing the biosimilarity of CT-P10 \& reference rituximab}

- In vitro structural, physicochemical, biological and functional analyses have demonstrated that CT-P10 and reference rituximab are highly similar.

- Phase I and III randomized controlled trials have demonstrated pharmacokinetic equivalence between CT-P10 and reference rituximab and comparable efficacy, safety, pharmacodynamics and immunogenicity in patients with rheumatoid arthritis.

- Pharmacokinetic equivalence of CT-P10 and reference rituximab and comparable safety, pharmacodynamics and immunogenicity have also been shown in patients with advanced follicular lymphoma.

\section{Scientific rationale for extrapolation of CT-P10 clinical data across indications}

- Rheumatoid arthritis was an appropriate indication in which to compare CT-P10 with reference rituximab as it was considered adequately sensitive to detect clinically meaningful differences in efficacy and safety between products.

- B cells play a primary role in the pathology of disease for all indications of reference rituximab. The ability of CT-P10 and reference rituximab to deplete B cells is highly comparable.

- Depletion of B cells by rituximab has been attributed to complement-dependent cytotoxicity, antibody-dependent cellular cytotoxicity and apoptosis.

- The contribution of these mechanisms across indications is uncertain. However, it has been demonstrated that CT-P10 and reference rituximab are comparable in their ability to induce these activities.

\section{Conclusion}

- Based on the totality of evidence, the EMA concluded that CT-P10 was highly similar to reference rituximab and that it was scientifically justified to approve the biosimilar for all indications held by the reference product.

\section{References}

Papers of special note have been highlighted as:

- of interest; $\bullet$ of considerable interest

1 European Medicines Agency. Guideline on similar biological medicinal products containing biotechnology-derived proteins as active substance: non-clinical and clinical issues.

www.ema.europa.eu/docs/en_GB/
document_library/Scientific_ guideline/2015/01/WC500180219.pdf

2 European Medicines Agency. Guideline on similar biological medicinal products containing biotechnology-derived proteins as active substance: quality issues (revision 1). www.ema.europa.eu/docs/en_GB/ document_library/Scientific_ guideline/2014/06/WC500167838.pdf
3 U.S. Food \& Drug Administration. Quality considerations in demonstrating biosimilarity of a therapeutic protein product to a reference product. Guidance for industry. www.fda.gov/ downloads/drugs/guidances/ucm291134.pdf

4 U.S. Food \& Drug Administration. Scientific considerations in demonstrating biosimilarity to a reference product. Guidance for industry. www.fda.gov/downloads/drugs/guidances/ ucm291128.pdf 
5 European Medicines Agency. Guideline on similar biological medicinal products containing monoclonal antibodies - nonclinical and clinical issues. www.ema.europa. eu/docs/en_GB/document_library/ Scientific_guideline/2012/06/ WC500128686.pdf

6 European Medicines Agency. Guideline on similar biological medicinal products. www. ema.europa.eu/docs/en_GB/document_ library/Scientific_guideline/2014/10/ WC500176768.pdf

7 European Medicines Agency. Concept paper on extrapolation of efficacy and safety in medicine development. www.ema.europa.eu/ docs/en_GB/document_library/Scientific_ guideline/2012/06/WC500129285.pdf

8 European Medicines Agency. Committee for Medicinal Products for Human Use. Assessment report for Truxima (procedure No. EMEA/H/C/004112/0000). www.ema. europa.eu/docs/en_GB/document_library/ EPAR_-_Public_assessment_report/ human/004112/WC500222695.pdf

-• Analytical, preclinical and clinical data supporting the approval of CT-P10 by the EMA. Includes in vitro data demonstrating similarity of CT-P10 and reference rituximab with respect to antibodydependent cell-mediated cytotoxicity, complement-dependent cytotoxicity and apoptosis-inducing activity.

9 Buske C, Ogura M, Kwon H-C, Yoon SW. An introduction to biosimilar cancer therapeutics: definitions, rationale for development and regulatory requirements. Future Oncol. 13(15s), 1-3, (2017).

10 Yoo D-H, Suh C-H, Shim SC et al. A multicentre randomised controlled trial to compare the pharmacokinetics, efficacy and safety of CT-P10 and innovator rituximab in patients with rheumatoid arthritis. Ann. Rheum. Dis. 76(3), 566-570 (2016).

- Pivotal clinical trial demonstrating pharmacokinetic equivalence and comparable efficacy, safety and immunogenicity of CT-P10 and reference rituximab in patients with rheumatoid arthritis. This study provided key data for determining biosimilarity and supporting cross-indication extrapolation.

11 Coiffier B, Sancho J, Jurczak W et al. Pharmacokinetic and safety of CT-P10, a biosimilar candidate to the rituximab reference product, in patients with newly diagnosed advanced stage follicular lymphoma (AFL). Blood 128(22), 1807 (2016).
- Key comparative clinical trial of CT-P10 and reference rituximab in patients with advanced follicular lymphoma, which supported extrapolation of CT-P10 in oncological indications. This study demonstrated pharmacokinetic equivalence and comparable pharmacodynamics, safety and immunogenicity between treatment groups.

12 Suh C-H, Berrocal Kasay A, Chalouhi El-Khouri E et al. Pharmacokinetics and safety of three formulations of rituximab (CT-P10, US-sourced innovator rituximab and EU-sourced innovator rituximab) in patients with rheumatoid arthritis: results from phase 3 randomized controlled trial over 24 weeks. Arthritis Rheumatol. 68(Suppl. 10), 2038-2040 (2016).

13 Yoo D-H, Majstorovic L, Kasay A et al. Efficacy and safety of CT-P10, rituximab biosimilar candidate, and innovator rituximab in patients with rheumatoid arthritis: results from Phase 3 randomized controlled trial over 24 weeks. Arthritis Rheumatol. 68(Suppl. 10), 1635 (2016).

14 Yoo D-H, Park W, Slawomir J et al. Impact of anti-drug antibody on efficacy and safety over week 24 in both CT-P10 and innovator rituximab treatment groups. Arthritis Rheumatol. 66, S663-S664 (2014).

15 Yoo D-H, Park W, Suh C-H et al. Efficacy and safety of switched CT-P10 from innovator rituximab compared with those of maintained CT-P10 in patients with rheumatoid arthritis up to 56 weeks. Arthritis Rheumatol. 67(Suppl. 10), 1675 (2015).

16 Yoo D-H, Park W, Suh C-H. Efficacy and safety of rituximab biosimilar candidate (CT- P10) and innovator rituximab in patients with rheumatoid arthritis: results from Phase I randomized controlled trial over 72 weeks. Arthritis Rheumatol. 67(Suppl. 10), 2058 (2015).

17 Mok CC. Rituximab for the treatment of rheumatoid arthritis: an update. Drug Des. Devel. Ther. 8, 87-100 (2014).

18 Bugatti S, Vitolo B, Caporali R. B cells in rheumatoid arthritis: from pathogenic players to disease biomarkers. Biomed. Res. Int. 2014(681678), 1-14 (2014).

19 Shaffer AL III, Young RM, Staudt LM. Pathogenesis of human B cell lymphomas. Annu. Rev. Immunol. 30, 565-610 (2012).

20 Samuels J, Ng Y-S, Coupillaud C, Paget D, Meffre E. Impaired early B cell tolerance in patients with rheumatoid arthritis. J. Exp. Med. 201(10), 1659-1667 (2005).
21 LeBien TW, Tedder TF. B lymphocytes: how they develop and function. Blood 112(5), 1570-1580 (2008).

22 Cartron G, Watier H, Golay J, Solal-Celigny P, Cartron G. From the bench to the bedside: ways to improve rituximab efficacy. Blood 104(9), 2635-2642 (2014).

- Review summarizing the mechanisms of action of rituximab and the potential for optimizing its use to improve clinical efficacy.

23 Press OW, Appelbaum F, Ledbetter JA et al. Monoclonal antibody 1F5 (anti-CD20) serotherapy of human B cell lymphomas. Blood 69(2), 584-591 (1987)

24 Boross P, Leusen JHW. Mechanisms of action of CD20 antibodies. Am. J. Cancer Res. 2(6), 676-690 (2012).

- Review of rituximab's mechanisms of action and the relative contributions of antibodydependent cell-mediated cytotoxicity, complement-dependent cytotoxicity and apoptosis to the drug's activity in vivo.

25 Oflazoglu E, Audoly LP. Evolution of anti-CD20 monoclonal antibody therapeutics in oncology. $m A$ bs 2(1), 14-19 (2010).

26 Cragg MS, Morgan SM, Chan HTC et al. Complement-mediated lysis by anti-CD20 $\mathrm{mAb}$ correlates with segregation into lipid rafts. Blood 101(3), 1045-1052 (2003).

- In vitro study reporting links between anti-CD20 monoclonal antibody activation of the complement pathway and redistribution of $\mathrm{CD} 20$ into lipid rafts.

27 Cragg MS, Glennie MJ. Antibody specificity controls in vivo effector mechanisms of anti-CD20 reagents. Blood 103(7), 27382743 (2004)

28 Golay J, Cittera E, Di Gaetano N et al. The role of complement in the therapeutic activity of rituximab in a murine B lymphoma model homing in lymph nodes. Haematologica 91(2), 176-183 (2006).

29 Di Gaetano N, Cittera E, Nota R et al. Complement activation determines the therapeutic activity of rituximab in vivo. J. Immunol. 171(3), 1581-1587 (2003).

30 Uchida J, Hamaguchi Y, Oliver JA et al. The innate mononuclear phagocyte network depletes B lymphocytes through $\mathrm{Fc}_{\mathrm{c}}$ receptor-dependent mechanisms during anti-CD20 antibody immunotherapy. J. Exp. Med. 199(12), 1659-1669 (2004).

31 Weng WK, Levy R. Expression of complement inhibitors CD46, CD55, and CD59 on tumor cells does not predict clinical outcome after rituximab treatment in 
follicular non-Hodgkin lymphoma. Blood 98(5), 1352-1357 (2001).

32 Bannerji R, Kitada S, Flinn IW et al. Apoptotic-regulatory and complementprotecting protein expression in chronic lymphocytic leukemia: relationship to in vivo rituximab resistance. J. Clin. Oncol. 21(8), 1466-1471 (2003).

33 Van Meerten T, Van Rijn RS, Hol S, Hagenbeek A, Ebeling SB. Complementinduced cell death by rituximab depends on CD20 expression level and acts complementary to antibody-dependent cellular cytotoxicity. Clin. Cancer Res. 12(13), 4027-4035 (2006).

34 Tedeschi A, Vismara E, Ricci F, Morra E, Montillo M. The spectrum of use of rituximab in chronic lymphocytic leukemia. Onco Targets Ther. 3, 227-246 (2010).

35 Rajasekaran N, Chester C, Yonezawa A, Zhao $\mathrm{X}$, Kohrt HE. Enhancement of antibodydependent cell mediated cytotoxicity: a new era in cancer treatment. Immunotargets Ther. 4, 91-100 (2015).

36 Dall'Ozzo S, Tartas S, Paintaud G et al. Rituximab-dependent cytotoxicity by natural killer cells: Influence of FCGR3a polymorphism on the concentration-effect relationship rituximab-dependent cytotoxicity by natural killer cells. Cancer Res. 64(13), 4664-4669 (2004).

37 Weng WK, Levy R. Two immunoglobulin G fragment $C$ receptor polymorphisms independently predict response to rituximab in patients with follicular lymphoma. J. Clin. Oncol. 21(21), 3940-3947 (2003).

Quartuccio L, Fabris M, Pontarini E et al. The 158VV Fcgamma receptor 3A genotype is associated with response to rituximab in rheumatoid arthritis: results of an Italian multicentre study. Ann. Rheum. Dis. 73(4), 716-721 (2014).

39 Dornan D, Spleiss O, Yeh RF et al. Effect of FCGR2A and FCGR3A variants on CLL outcome. Blood 116(20), 4212-4222 (2010).

Ghesquières H, Cartron G, Seymour JF et al. Clinical outcome of patients with follicular lymphoma receiving chemoimmunotherapy in the PRIMA study is not affected by FCGR3A. Blood 120(13), 2650-2657 (2012).

41 Liu F, Ding H, Jin X et al. FCGR3A 158V/F polymorphism and response to frontline R-CHOP therapy in diffuse large B-cell lymphoma. DNA Cell Biol. 33(9), 616-623 (2014).
42 Bonavida B. Rituximab-induced inhibition of antiapoptotic cell survival pathways: implications in chemo/immunoresistance, rituximab unresponsiveness, prognostic and novel therapeutic interventions. Oncogene 26(25), 3629-3636 (2007).

43 Pedersen IM, Buhl AM, Klausen P, Geisler $\mathrm{CH}$, Jurlander J. The chimeric anti-CD20 antibody rituximab induces apoptosis in B-cell chronic lymphocytic leukemia cells through a p38 mitogen activated proteinkinase-dependent mechanism. Blood 99(4), 1314-1319 (2002).

44 Jazirehi AR, Huerta-Yepez S, Cheng G, Bonavida B. Rituximab (chimeric anti-CD20 monoclonal antibody) inhibits the constitutive nuclear factor-\{kappa\}B signaling pathway in non-Hodgkin's lymphoma B-cell lines: role in sensitization to chemotherapeutic drug-induced apoptosis. Cancer Res. 65(1), 264-276 (2005).

45 Vega MI, Huerta-Yepez S, Martinez-Paniagua $\mathrm{M}$ et al. Rituximab-mediated cell signaling and chemo/immuno-sensitization of drug-resistant B-NHL is independent of its $\mathrm{Fc}$ functions. Clin. Cancer. Res. 15(21), 6582-6594 (2009). 
